\title{
Are interleukin-15 and -22 a new pathogenic factor in pustular palmoplantar psoriasis?
}

\author{
Aleksandra Lesiak르, Igor Bednarski², Marta Pałczyńska², Ewelina Kumiszcza², Marzena Kraska-Gacka', \\ Anna Woźniacka ${ }^{1}$, Joanna Narbutt ${ }^{1}$
}

${ }^{1}$ Department of Dermatology, Medical University of Lodz, Lodz, Poland

${ }^{2}$ Student Research Group at the Department of Dermatology, Medical University of Lodz, Lodz, Poland

Adv Dermatol Allergol 2016; XXXIII (5): 336-339

DOI: $10.5114 / a d a .2016 .62838$

\begin{abstract}
Introduction: Pustular palmoplantar psoriasis (PPP) is a rare type of psoriasis affecting mainly distal parts of the limbs. Despite numerous theories about etiology of PPP, the pathogenesis still remains unclear. Recent data indicate that interleukin (IL)-15, IL-17 and IL-22 enhance a proinflammatory response in certain skin inflammatory diseases such as psoriasis and atopic dermatitis. There is also evidence that anti-endomysial (anti-EMA) and anti-gliadin (AGA) antibodies are engaged in PPP development.

Aim: To assess IL-15, IL-17, IL-22 serum levels and evaluate the presence of anti-endomysial and anti-gliadin antibodies in patients with PPP.

Material and methods: The study group consisted of 20 females of the mean age of 51.8 suffering from PPP. Additionally 29 healthy individuals, age and sex matched, served as controls. ELISA was performed to evaluate serum IL-15, IL-17, IL-22 concentrations while an indirect immunofluorescence test (IIF) was used to determine anti-EMA and AGA presence.

Results: The mean value of IL-15 and IL-22 serum concentrations was significantly higher in the study group than in the control group (IL-15: 6.48 vs. 4.88 pg/ml; IL-22: 81.47 vs. 4.90 pg/ml, respectively; $p<0.05$ for all comparisons). The IL-17 serum level in the study group was higher when compared to the control group ( $2.0 \mathrm{vs} .0 .75 \mathrm{pg} / \mathrm{ml})$, however the results were not statistically significant $(p=0.26)$. There were no anti-EMA and AGA antibodies detected, both in the control and study group.
\end{abstract}

Conclusions: The results obtained may suggest involvement of IL-15 and IL-22 in the pathogenesis of PPP.

Key words: interleukin 15, interleukin 17, interleukin 22, anti-endomysial antibodies, anti-gliadin antibodies, pustular palmoplantar psoriasis.

\section{Introduction}

Plaque psoriasis is a chronic and recurrent inflammatory skin disease affecting 2-3\% of the European population [1, 2]. Pustular palmoplantar psoriasis (PPP) is a less frequent type of psoriasis with different genetic background [3]. Typical signs of PPP include sterile pustules located on the red skin affecting palms and soles. Skin lesions are painful and rarely disseminated. The prevalence of PPP, like other immune-mediated diseases is much more frequent in the female population [4]. It is suspected that intensity of skin lesions positively correlates with serum anti-gliadin antibody (AGA) and anti-transglutaminase antibody (TGA) levels [5, 6]. The relation between psoriasis and other immune-mediated diseases like rheumatoid arthritis and inflammatory bowel diseases is well established in the literature. In the pathogenesis of psoriasis, immunological factors are playing a key role. An increased serum level of several proinflammatory cytokines, such as interleukin (IL)-15 [7], IL-17 [8] and IL-22 [9] was found in certain studies. There is increasing evidence that IL-17 regulates inflammatory response in psoriasis, induces production and release of several cytokines, such as IL-6, granulocyte-colony stimulating factor (G-CSF), $\mathrm{IL}-1 \beta$, transforming growth factor (TGF) $\beta$ and tumor necrosis factor (TNF)- $\alpha$, chemokines (IL-8, GRO- $\alpha$, and MCP-1) and prostaglandins from fibroblasts, endothelial cells, keratinocytes, epithelial cells and macrophages [10, 11]. Interleukin 17 serum concentration is increased in many autoimmune diseases, for example rheumatoid arthritis, lupus erythe-

Address for correspondence: Assoc. Prof. Aleksandra Lesiak, Department of Dermatology, Medical University of Lodz, Plac Hallera 1, 90-647 Lodz, Poland, phone/fax: +48 426867 981, +48 426884 565, e-mail: aleksandra.lesiak@umed.lodz.pl Received: 14.07.2015, accepted: 23.10.2015. 
matosus, asthma, transplant rejection and in psoriasis [12]. Interleukin 22 stimulates proliferation of keratinocytes. The interaction between IL-22 and IL-17 results in activation of proinflammatory cytokines and chemokines and increases vascular endothelial growth factor (VEGF) production from keratinocytes [13]. Interleukin 15 participates in chronic inflammatory response, promotes leukocyte growth and is a potent inhibitor of lymphocyte apoptosis [14].

\section{Aim}

Few data on the pathogenesis of pustular palmoplantar psoriasis, especially proinflammatory serum cytokine levels and presence of specific autoantibodies were the reason why we aimed to assess certain immunological factors in patients with PPP [15].

\section{Material and methods}

\section{Study group}

The study consisted of 20 female patients of the mean age of 51.8 (range: 50-70 years old), suffering from pustular palmoplantar psoriasis, which was confirmed by histological examination. We decided to perform the study only on females, because of a higher prevalence of PPP in females and to eliminate sex-related bias. Additionally 29 healthy individuals, age- and sex-matched, served as the controls. Patients were included into the study based on the physical examination and medical history. Patients who were receiving topical treatment in the past 2 weeks and systemic treatment in the past 2 months, pregnant women and individuals with concomitant diseases were excluded from the study. Signed informed consent was taken from all the patients and controls before enrollment to the study.

\section{Methods}

Blood samples were collected in order to assay the serum concentration of IL-15, IL-17, IL-22, anti-endomysi- al and anti-gliadin IgA antibodies. All the samples were taken in the active stage of disease. ELISA was performed to evaluate serum IL-15, IL-17, IL-22 concentrations (R\&D Systems Europe Ltd. Abingdon, United Kingdom) while an indirect immunofluorescence test (IIF) was used to determine EMA and AGA levels (Euroimmun Medizinische Labordiagnostika AG, Luebeck, Germany).

\section{Statistical analysis}

For statistical analysis the Shapiro-Wilk test and Mann-Whitney $U$ test were applied, and arithmetic mean, standard deviation, median, lower and upper quartile were calculated. To measure the possible statistical relation between two variables, Spearman's rank correlation coefficient was used. Values of $p<0.05$ were considered statistically significant.

\section{Results}

The serum concentration of all cytokines was higher in the study group in comparison to healthy controls. The IL-15 serum concentration mean and median values were notably higher in the study group $(6.48 \pm 2.82$, median: $5.95)$ than in the control group (4.88 \pm 0.6 , median: 4.86 , $p=0.000137$ for all comparisons). The same tendency was observed in IL-22 assay results ( $81.47 \pm 175.24$ and median 26.35 in the study group vs. $4.9 \pm 4.44$ and median 3.79 in the control group, $p=0.000001$ for all comparisons). The concentration of IL-17 in the study group (2.0 \pm 4.13 and median 0.0), compared to the control group ( $0.75 \pm 1.72$ and median 0.0$)$ was higher, however the results were not statistically significant ( $p=0.41$, for all comparisons). All interleukin concentration values are expressed in $\mathrm{pg} / \mathrm{ml}$. There were no anti-endomysial (EMA) and anti-gliadin (AGA) antibodies detected, both in the control and study group. Detailed results are shown in Table 1, Figures 1 and 2.

Table 1. Interleukins 1, 17 and 22 detailed results in investigated groups

\begin{tabular}{|c|c|c|c|c|c|c|}
\hline \multirow[t]{2}{*}{ Parameter } & \multicolumn{2}{|c|}{ IL-15 assay results [pg/ml] } & \multicolumn{2}{|c|}{ IL-17 assay results $[\mathrm{pg} / \mathrm{ml}]$} & \multicolumn{2}{|c|}{ IL-22 assay results [pg/ml] } \\
\hline & Study group & Control group & Study group & Control group & Study group & Control group \\
\hline Valid observations & 20 & 29 & 20 & 29 & 20 & 29 \\
\hline Mean value & 6.48 & 4.88 & 2.0 & 0.75 & 81.47 & 4.9 \\
\hline Median & 5.95 & 4.86 & 0.00 & 0.00 & 26.35 & 3.79 \\
\hline Minimal value & 4.42 & 3.98 & 0.00 & 0.00 & 2.5 & 0 \\
\hline Maximal value & 17.74 & 6.28 & 17.07 & 5.86 & 740.01 & 17.32 \\
\hline Lower quartile & 5.03 & 4.42 & 0.00 & 0.00 & 12.17 & 2.5 \\
\hline Upper quartile & 6.60 & 5.18 & 3.37 & 0.00 & 51.5 & 5.08 \\
\hline Standard deviation & 2.82 & 0.60 & 4.13 & 1.72 & 175.24 & 4.44 \\
\hline $\begin{array}{l}\text { Statistical significance } \\
\text { (Mann-Whitney } U \text { test) }\end{array}$ & \multicolumn{2}{|c|}{$p=0.000137$} & \multicolumn{2}{|c|}{$p=0.41$} & \multicolumn{2}{|c|}{$p=0.000001$} \\
\hline
\end{tabular}




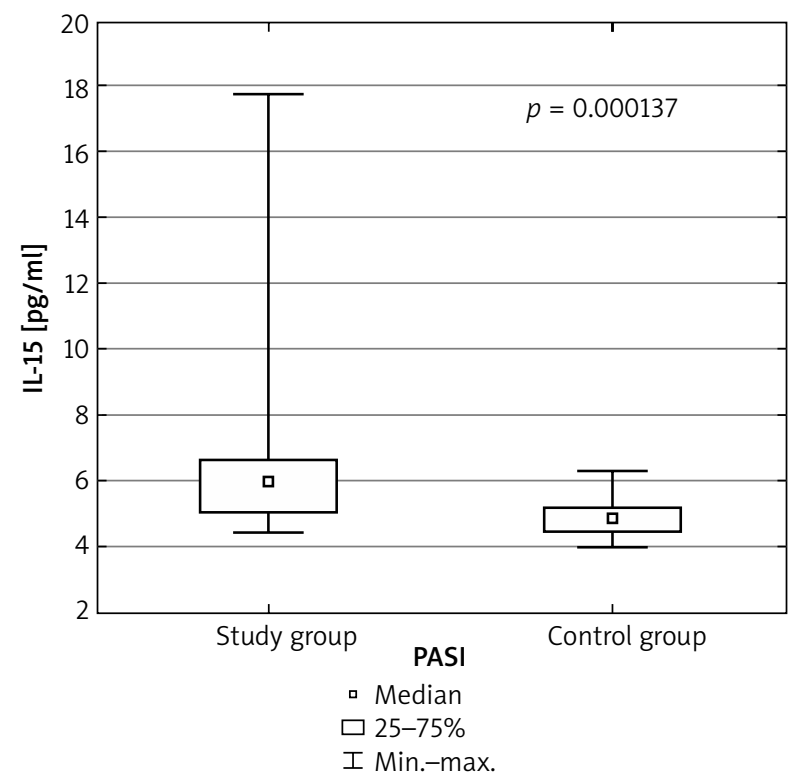

Figure 1. Serum concentration of IL-15 in the study and control group. Mann-Whitney $U$ test was performed to evaluate differences between the control and study group. Box-plot shows mean, median, upper and lower quartile, minimum and maximum values

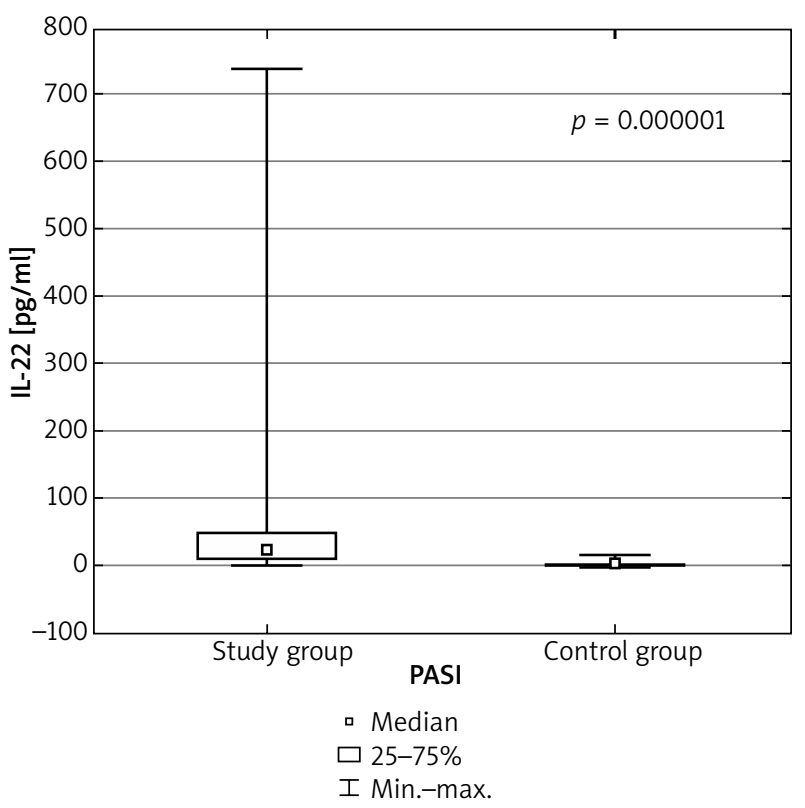

Figure 2. Serum concentration of IL-22 in the study and control group. Mann-Whitney $U$ test was performed to evaluate differences between the control and study group. Box-plot shows mean, median, upper and lower quartile, minimum and maximum values

\section{Discussion}

The role of IL-15, IL-17, IL-22, AGA and anti-EMA antibodies has been hardly explored in the context of pustular palmoplantar psoriasis. Most of the publications concerned the relation between these cytokines and prevalence of psoriasis vulgaris and generalized psoriasis pustulosa (GPP), so we could compare our results only with these data.

Interleukin 15 is secreted by mononuclear phagocytes, structurally similar to IL-2 and leads to proliferation of NK-cells, activation of T-cells and induces TNF- $\alpha$ and IL-17 production [16, 17]. Interleukin 15 influences angiogenesis, neutrophil and macrophage recruitment and acanthosis [16]. It is proven that dysregulation of IL-15 expression is occurring in patients with rheumatoid arthritis, inflammatory bowel diseases, coeliac disease and psoriasis. In our study, we revealed for the first time the higher serum concentration of IL-15 in PPP patients, what suggests the pathogenic role of this cytokine in the entity development. Interleukin 17 is an interleukin produced by Th-17 cells, which were identified as CD4-positive T-cell subset implicated in the pathogenesis of psoriasis [18]. It is involved in the proinflammatory response, stimulating the production of many other cytokines (IL-6, G-CSF, IL-1 $\beta$, TGF- $\beta$ and TNF- $\alpha$ ), chemokines (IL-8, GRO- $\alpha$, and MCP-1) and prostaglandins $[10,19]$.

Our study revealed a difference in IL-17 levels between patients and controls, but it turned out to be statistically insignificant. In the research carried out by Yilmaz et al. [19], the IL-17 levels in patients with pustular psoriasis were significantly higher than in the control group and other subgroups (guttate psoriasis and plaque psoriasis). Results obtained by Kakeda et al. [8] were similar, revealing differences in IL-17A/F expression between GPP patients and the control group. Moreover, neutrophils were identified as the main source of IL-17A/F in pustules. Six cases of GPP analyzed by Yamamoto et al. [15] suggested a positive correlation between IL-17 levels and GPP score. Although we found no statistically relevant differences in the IL-17 serum level between the patients and the controls, we may assume that its higher concentration in the patients points out its potential pathogenic role. Interleukin 17 is becoming also a novel therapeutic aim in psoriasis treatment. A clinical trial conducted with the use of secukinumab, a monoclonal antibody against IL-17A, revealed normalization of skin histology and regression of skin lesions, indirectly proving involvement of IL-17 in psoriasis [20].

Interleukin 22 cytokine is produced by Th17 cells together with IL-17, enhancing the proinflammatory response and stimulating the proliferation of the keratinocytes [9, 13]. It is involved in a variety of skin inflammatory processes, such as psoriasis and atopic dermatitis [13, 21].

We revealed the elevated IL-22 serum level in the patients. This result corresponds with the publication by Yamamoto et al. [15] in which the IL-22 serum level correlated with the activity of generalized pustular psoriasis (GPP), white blood cells counts (WBC) and C-reactive protein (CRP) levels. Moreover, it decreased after treatment in parallel with the GPP score [15]. This observation was also made by Shimauchi et al. [9] who showed elevated IL-22 along with 
VEGF and correlation with PASI score and a significant IL-22 decrease after biological treatment. Our results showed for the first time the role of IL-22 in PPP development.

Due to a common pathogenic background of coeliac disease (CD) and psoriasis, a possible relationship between these two diseases has been suggested [22]. There have been publications confirming a higher prevalence of antibodies characteristic of CD among psoriatic patients, mostly anti-gliadin immunoglobulin, anti-endomysial IgA antibody, anti-transglutaminase IgA antibody and antireticulin antibodies. The correlation between AGA serum expression and duration of psoriasis was pointed out. It also seemed to be more characteristic of patients with a stationary course of the disease [23]. We focused on anti-gliadin and anti-endomysial antibodies, but they were absent in both patients and control group. Research concerning Italian [24] and Kashmir population [25] suggests that there may be some genetic differences between populations as in these studies no correlations between AGA and the prevalence of psoriasis were found.

\section{Conclusions}

Based on our results we may conclude a potential role of IL-15 and IL-22 in the development of pustular palmoplantar psoriasis in Polish women patients. To our knowledge, this observation is made for the first time in the literature. We hope that in the near future new investigations on the larger study population will bring more details and lead to invention of new therapeutic regimens.

\section{Acknowledgments}

This study was funded by the National Center of Science, grant No. UMO-2013/11/B/NZ5/00037 and Medical University of Lodz project No. 503/1-152-01/503-11-002.

\section{Conflict of interest}

The authors declare no conflict of interest.

\section{References}

1. Schäfer T. Epidemiology of psoriasis: review and the German perspective. Dermatology 2006; 212: 327-37.

2. Kagami S, Rizzo HL, Lee JJ, et al. Circulating Th17, Th22, and Th1 cells are increased in psoriasis. J Invest Dermatol 2010; 130: 1373-83.

3. De Waal AC, van de Kerkhof PCM. Pustulosis palmoplantaris is a disease distinct from psoriasis. J Dermatolog Treat 2011; 22: 102-5.

4. Schon M, Boehncke WH. Psoriasis. N Engl J Med 2005; 352: 1899-912.

5. Woo WK, McMillan SA, Watson RGP, et al. Coeliac diseaseassociated antibodies correlate with psoriasis activity. $\mathrm{Br}$ J Dermatol 2004; 151: 891-4.

6. Humbert P, Pelletier F, Dreno B, et al. Gluten intolerance and skin diseases. Eur J Dermatol 2006; 16: 4-11.
7. Waldmann T. The biology of IL-15: implications for cancer therapy and the treatment of autoimmune disorders. J Investig Dermatol Symp Proc 2013; 16: S28-30.

8. Kakeda M, Schlapbach C, Danelon G, et al. Innate immune cells express IL-17A/F in acute generalized exanthematous pustulosis and generalized pustular psoriasis. Arch Dermatol Res 2014; 306: 933-8.

9. Shimauchi T, Hirakawa S, Suzuki T, et al. Serum interleukin-22 and vascular endothelial growth factor serve as sensitive biomarkers but not as predictors of therapeutic response to biologics in patients with psoriasis. J Dermatol 2013; 40: 805-12.

10. Furuzawa-Carballeda J, Vargas-Rojas MI, Cabral AR. Autoimmune inflammation from the Th17 perspective. Autoimmun Rev 2007; 6: 169-75.

11. Kolls JK, Lindén A. Interleukin-17 family members and inflammation. Immunity 2004; 21: 467-76.

12. Aggarwal S, Gurney AL IL-17: prototype member of an emerging cytokine family. J Leukoc Biol 2002; 71: 1-8.

13. Koga C, Kabashima K, Shiraishi N, et al. Possible pathogenic role of Th17 cells for atopic dermatitis. J Invest Dermatol 2008; 128: 2625-30.

14. Rückert R, Asadullah K, Seifert M, et al. Inhibition of keratinocyte apoptosis by IL-15: a new parameter in the pathogenesis of psoriasis? J Immunol 2000; 165: 2240-50.

15. Yamamoto M, Imai Y, Sakaguchi Y, et al. Serum cytokines correlated with the disease severity of generalized pustular psoriasis. Dis Markers 2013; 34: 153-61.

16. Michalak-Stoma A, Pietrzak A, Szepietowski JC, et al. Cytokine network in psoriasis revisited. Eur Cytokine Netw 2011; 22: 160-8.

17. Gardiner CM, Dunphy S. NK cells and psoriasis. J Biomed Biotechnol 2011; 2011: 248317.

18. Blauvelt A. T-helper 17 cells in psoriatic plaques and additional genetic links between IL-23 and psoriasis. I Invest Dermatol 2008; 128: 1064-7.

19. Yilmaz SB, Cicek N, Coskun M, et al. Serum and tissue levels of IL-17 in different clinical subtypes of psoriasis. Arch Dermatol Res 2012; 304: 465-9.

20. U.S. Food and Drug Administration. Secukinumab (AIN457): Advisory Commitee Briefing Material: Available for public release. Prepared by Novartis Pharmaceuticals Corporation for the Dermatologic and Ophthalmic Drugs Advisory Committee Meeting 2014. Available from: http://www.fda.gov/downloads/AdvisoryCommittees/CommitteesMeetingMaterials/ Drugs/DermatologicandOphthalmicDrugsAdvisoryCommittee/UCM419023.pdf.

21. Zaba LC, Fuentes-Duculan J, Eungdamrong NJ, et al. Psoriasis is characterized by accumulation of immunostimulatory and Th1/Th17 cell-polarizing myeloid dendritic cells. J Invest Dermatol 2009; 129: 79-88.

22. Akbulut S, Gür G, Topal F, et al. Coeliac disease-associated antibodies in psoriasis. Ann Dermatol 2013; 25: 298-303.

23. Nagui N, El Nabarawy E, Mahgoub D, et al. Estimation of (IgA) anti-gliadin, anti-endomysium and tissue transglutaminase in the serum of patients with psoriasis. Clin Exp Dermatol 2011; 36: 302-4.

24. De Bastiani R, Gabrielli M, Lora L, et al. Association between coeliac disease and psoriasis: Italian Primary Care Multicentre Study. Dermatology 2015; 230: 156-60.

25. Sultan SJ, Ahmad QM, Sultan ST. Antigliadin antibodies in psoriasis. Australas I Dermatol 2010; 51: 238-42. 